\title{
Stress-related differential expression of multiple $\beta$-carotene ketolase genes in the unicellular green alga Haematococcus pluvialis
}

Jun-Chao Huang ${ }^{\mathrm{a}}$, Feng Chen ${ }^{\mathrm{a}^{*}}$, Gerhard Sandmann ${ }^{\mathrm{b}^{*}}$

${ }^{a}$ Department of Botany, The University of Hong Kong, Pokfulam Road, Hong Kong, P.R. China

${ }^{b}$ Botanical Institute, Goethe University Frankfurt, P.O. Box 111932, D-60054 Frankfurt, Germany

* Corresponding authors. Tel: +852 22990309; fax: +856 22990311.

E-mail address: sfchen@hkusua.hku.hk (F.Chen).

Tel: +49 69 79824746; fax: +49 6979824822 .

E-mail address: sandmann@em.uni-frankfurt.de (G. Sandmann) 


\section{ABSTRACT}

The unicellular green alga Haematococcus pluvialis accumulates large amounts of ketocarotenoid astaxanthin under a variety of environmental stresses. Here we report the identification and expression of three different $\beta$-carotene ketolase genes $(b k t)$ that are involved in the biosynthesis of astaxanthin in a single strain of the alga. Bkt1 and bkt2 proved to be the crtO and bkt previously isolated from two different strains of $H$. pluvialis. Bkt3 is a novel third one which shared 95\% identical nucleotide sequence with bkt2. Nitrogen deficiency alone could not induce the alga cells to produce astaxanthin in three days even though it enhances the expression of the bkt genes to 3 times of that in normal growing cells within $16 \mathrm{~h}$. High light irradiation $\left(125 \mu \mathrm{mol} \mathrm{m} \mathrm{m}^{-2} \mathrm{~s}^{-1}\right)$ or $45 \mathrm{mM}$ sodium acetate greatly increased the expression of $b k t$ genes to 18 or 52 times of that in normal growing cells, resulting in an accumulation of substantial astaxanthin (about $6 \mathrm{mg} / \mathrm{g}$ dry biomass) in three days. It is suggested that the existence of the multiple $b k t$ genes and their strong up-regulation by different stress conditions is one of the reasons that $H$. pluvialis accumulates large amounts of astaxanthin in an instant response to stress environments.

Key-words: astaxanthin; $\beta$-carotene ketolase; Haematococcus pluvialis; real-time PCR 


\section{Introduction}

Haematococcus pluvialis accumulates large amounts of ketocarotenoid astaxanthin in response to high light irradiation, nitrogen limitation and salt stress (Yong and Lee, 1991; Kobayashi et al., 1992; Boussiba, 2000). Diverse functions of astaxantin in $H$. pluvialis related to its antioxidative properties have been proposed (Hagen et al., 1993; Kobayashi et al., 1997). This green microalga is the main natural source of astaxanthin as feed supplements in the farming of trout and salmon (Meyers, 1994; Johnson and Schroeder, 1996). It is also an ideal organism to study the regulation of ketocarotenoid synthesis (Sun et al., 1998; Linden, 1999; Boussiba, 2000; Grünewald et al., 2000; Grünewald et al., 2001; Steinbrenner and Linden, 2001, 2003). Higher plants and green algae share the same carotenoid biosynthetic pathway to $\beta$-carotene (Cunningham and Gantt, 1998). In H. pluvialis the specific steps leading to astaxanthin are further catalyzed by $\beta$-carotene ketolase (Kajiwara et al., 1995; Lotan and Hirschberg, 1995) and $\beta$-carotene hydroxylase (Linden, 1999). The $\beta$-carotene ketolase is the only enzyme that exclusively participates in the secondary carotenoid pathway to astaxanthin in Haematococcus. This enzyme plays an essential role in stress-dependent initiation of astaxanthin synthesis. Two different $\beta$-carotene ketolase cDNAs bkt1* (formerly crtO (Lotan and Hirschberg, 1995)) and bkt2 (formerly bkt (Kajiwara et al., 1995)) were isolated from two different strains of $H$. pluvialis on different induced conditions. It is still unknown whether this indicates the existence of multiple carotene ketolases in Haematococcus (Sun et al., 1998), or only reflects strain differences (Grünewald et al., 2000). Therefore it is important to know about the nature of the ketolase genes and the relationship between the regulation of these genes and the accumulation of astaxanthin in Haematococcus when the algal cells are undergone different stress conditions. 
In the present study, we focus on the nature of bkt genes, their differential expression and astaxanthin accumulation in $H$. pluvialis Flotow NIES-144 in response to different stress conditions.

*Foot note: All $\beta$-carotene ketolase genes were designated bkt as introduced in Misawa et al. (1995b). It uses 3 letters indicating their function according to the nomenclature of carotenogenic genes from eukaryotes. Numbers were given in the order of publication.

\section{Materials and methods}

\subsection{Haematococcus pluvialis and growth conditions}

H. pluvialis Flotow NIES-144 was obtained from the National Institute for Environmental Studies (Tsukuba, Japan). H. pluvialis was grown in $250 \mathrm{ml}$ Erlenmeyer flasks containing a medium as described by Kobayashi et al. (Kobayashi et al., 1993). For control samples, sodium acetate $(14.8 \mathrm{mM})$ was removed from the medium. Cells were grown at $20^{\circ} \mathrm{C}$ under a dark/light cycle of $12 \mathrm{~h}$ of illumination at $20 \mu \mathrm{mol} \mathrm{m} \mathrm{m}^{-2} \mathrm{~s}^{-1}$ and $12 \mathrm{~h}$ of darkness for 5 days. For induction of astaxanthin biosynthesis, sodium acetate or/and $\mathrm{FeSO}_{4}$ were used at final concentrations of 45 $\mathrm{mM}$ and $450 \mu \mathrm{M}$, respectively, and sodium chloride at $100 \mathrm{mM}$. For nitrogen deficiency experiment, cells grown mixotrophically were harvested, washed with sterile deionized water and resuspended in a TAP medium (Gorman and Levine, 1966) without nitrogen source and sodium acetate. For high light treatment, cultures of $H$. pluvialis were illuminated at $125 \mu \mathrm{mol} \mathrm{m} \mathrm{m}^{-2}$. 


\subsection{DNA and RNA extraction}

DNA and RNA techniques were followed according to the standard methods (Sambrook et al., 1989). DNA was extracted using a modified CTAB method (Stewart and Via, 1993). RNA was isolated from aliquots of about $10^{7}$ cells harvested after $5 \mathrm{~d}$ of growth and $16 \mathrm{~h}$ of varying inductions using TRI REAGENT ${ }^{\circledR}$ (Molecular Research Center, Ohio) according to the manufacturer's instructions. The concentration of total DNA and RNA was determined spectrophotometrically at $260 \mathrm{~nm}$.

\subsection{PCR, RT-PCR, and 3'RACE}

The sequences of $b k t 1$ (Genbank accession $\underline{\text { X86782})}$ ) and bkt2 (Genbank accession D45881) were aligned with Clustal X. The divergent 3' ends of the cDNA were selected as targets of PCR amplification. Primers were designed with the computer software Genetool. Primer sets and PCR product characteristics are listed in Table 1. Detection of bkt genes in H. pluvialis was performed by conventional PCR $\left(35\right.$ cycles of $94{ }^{\circ} \mathrm{C}$ for $20 \mathrm{~s}, 61{ }^{\circ} \mathrm{C}$ for $20 \mathrm{~s}, 72{ }^{\circ} \mathrm{C}$ for $30 \mathrm{~s}$ ). RT-PCR was performed with a one-step RT-PCR kit (Invitrogen) following the manufacturer's procedure. Forty ng of total RNA was used in a $25 \mu 1$ reaction volume. As an internal control for the relative amount of RNA, a fragment of actin gene was amplified with primers (forward: 5'AGCGGGAGATAGTGCGGGACA; reverse: 5'-ATGCCCACCGCCTCCATGC) designed from a cloned fragment of the actin gene from $H$. pluvialis (unpublished data). cDNA was synthesized at $55^{\circ} \mathrm{C}$ for 15 min followed with PCR amplification (32 cycles (for bkt genes) or 22 cycles (for actin gene) of $94{ }^{\circ} \mathrm{C}$ for $20 \mathrm{~s}, 61{ }^{\circ} \mathrm{C}$ for $20 \mathrm{~s}, 72{ }^{\circ} \mathrm{C}$ for $1 \mathrm{~min}$ ). 3 ' RACE of bkt was performed using a 5'/3' RACE kit (Roche molecular biochemicals, Germany). Primers F1, F2, and F3 (Table 1) were used for PCR amplification of dA-tailed cDNA and a nested PCR. The final PCR 
amplification product of 3'RACE was purified and cloned into pTZ57R/T vector (MBI Fermentas).

\subsection{Southern blot}

Eight micrograms of genomic DNA was digested with SacI or KpnI and SalI, three restriction enzymes without cutting site in the probed regions of the ketolase genes. The digested DNA was separated with a $0.8 \%$ agarose gel, transferred to a positively charged nylon membrane (Boehringer Mannheim/Roche), and hybridized with Dig-labeled DNA probes in the presence of $50 \%(\mathrm{v} / \mathrm{v})$ formamide at $47{ }^{\circ} \mathrm{C}$ for $16 \mathrm{hs}$. DNA probes were prepared by amplifying a $1 \mathrm{~kb}$ fragment from plasmid containing bkt 2 gene with a pair of primers (Table 1, Fp and Rp) (30 cycles of $94{ }^{\circ} \mathrm{C}$ for $20 \mathrm{~s}, 60{ }^{\circ} \mathrm{C}$ for $20 \mathrm{~s}, 72^{\circ} \mathrm{C}$ for $1 \mathrm{~min}$ ). Probe labeling and hybridization were carried out according to the instructions in the DIG Nonradioative Nucleic Acid Labeling and Detection System (Boehringer Mannheim/Roche). After hybridization, the membrane was washed twice with $0.1 \times \mathrm{SSC}$ containing $0.1 \% \mathrm{SDS}$ at $68{ }^{\circ} \mathrm{C}$ for $15 \mathrm{~min}$.

\subsection{DNA sequencing}

The nucleotide sequences of bkt genes derived from 3'RACE and RT-PCR were determined for both strands using the ABI prism Dye terminator cycle sequencing ready reaction kit (PerkinElmer Applied Biosystems).

\subsection{Functional analysis of bkt3 in E. coli}

The open reading frame of $b k t 3$ was PCR amplified and cloned into the vector pBluescript II $\mathrm{KS}$ as an in-frame fusion to the $\mathrm{lacZ}$ gene resulting in plasmid pHPb3. E. coli strain JM109 was used as a host for complementation experiments by co-transformation of $\mathrm{pHPb} 3$ with plasmids either pACCAR16 $\Delta \mathrm{crtX}$ or pACCAR25 $\Delta \mathrm{crtX}$ that harbor the carotenoid biosynthesis genes for 
producing $\beta$-carotene or zeaxanthin, respectively (Misawa et al., 1990). Carotenoids in the transformants were extracted and analyzed by HPLC according to Yuan and Chen (1998).

\subsection{Reverse Transcription and Real-Time PCR}

Reverse transcription was performed using SuperScript ${ }^{\mathrm{TM}}$ III First-Strand Synthesis System for RT-PCR (Invitrogen) according to the manufacturer's instruction. Real-time PCR was performed using a Rotor-Gene-2072 cycler (Corbett Research, Australia). Amplification was performed in Platimum ${ }^{\circledR} \mathrm{SYBR}^{\circledR}$ Green qPCR Supermix UDG (Invitrogen), specific primers at $0.5 \mu \mathrm{M}$ and cDNA of $36 \mathrm{ng}$ total RNA in a reaction volume of $20 \mu \mathrm{l}$. Plasmids containing the cDNA of $b k t 1$, or $b k t 3$ were serially diluted $\left(10^{-1}-10^{-7}\right.$ fmol) to generate a standard curve for each gene. The house-keeping gene actin was used as an internal standard for normalization of target RNA and RT reaction. The thermocycling program consisted of: hold at $50{ }^{\circ} \mathrm{C}$ for $5 \mathrm{~min}$, denaturation at $94{ }^{\circ} \mathrm{C}$ for $2 \mathrm{~min}$ followed by 40 cycles of PCR $\left(94{ }^{\circ} \mathrm{C}\right.$ for $15 \mathrm{~s}, 58{ }^{\circ} \mathrm{C}$ for $15 \mathrm{~s}, 68$ ${ }^{\circ} \mathrm{C}$ for $\left.30 \mathrm{~s}\right)$.

\subsection{Carotenoid extraction and HPLC analysis}

Carotenoids were extracted and analyzed according to Yuan and Chen (1998). Algal and E. coli cells were collected by centrifugation and freeze-dried. Extraction was carried out with a mixture of dichloromethane and methanol (25:75, v/v) until the cell debris was almost colorless. The combined extracts were evaporated to dryness and separated on a $5 \mu \mathrm{m}$ ODS $24.6 \times 250 \mathrm{~mm}$ analytical column (Waters Spherisorb ${ }^{\circledR}$ ) with a Waters high-performance liquid chromatograph. Individual carotenoids were identified by absorption spectra and their typical retention times compared to standard samples of pure carotenoids. Quantification of astaxanthin from $H$. pluvialis was carried out by area calibration with an authentic standard. 


\section{Results}

\subsection{Detection of bkt genes in H. pluvialis}

To detect whether $H$. pluvialis NISE 144 contains both $b k t 1$ and bkt2, genomic PCR was performed. The forward primer (F1, see Table 1) could base pair with both bkt1 and bkt2, while the reverse primer $\mathrm{R} 1$ is specific to $b k t 1$ and $\mathrm{R} 2$ specific to $b k t 2$. According to the sequences of $b k t 2$, PCR with primers F1 and R2 and genomic DNA of $H$. pluvialis NISE 144 would generate a $348 \mathrm{bp}$ fragment. If the alga also contains bkt1, an about $500 \mathrm{bp}$ fragment would be amplified with primers F1 and R1. PCR amplification with the specific primers F1 + R1/R2 did generate both 348 and $500 \mathrm{bp}$ fragments, indicating the possibility that $H$. pluvialis NISE 144 contains both $b k t 1$ and $b k t 2$ (Fig. 1). Furthermore this alga may contain one more bkt gene as indicated by the presence of an additional fragment (about $370 \mathrm{bp}$ ) in the genomic PCR product of bkt2 (Fig. 1, lane 2). Therefore we performed Southern blot to reveal the number of bkt gene in the genome of the alga.

H. pluvialis genomic DNA was digested with different restriction enzymes and subjected to Southern blot analysis. Using a $1 \mathrm{~kb}$ fragment of $b k t 2$ as a probe, the homologous fragments showed strong hybridization signals (Fig. 2). Each of the two separate digests showed three bands, suggesting the presence of three $b k t$ gene in the haploid genome of $H$. pluvialis. To verify this result and isolate the third bkt gene, 3' RACE of the bkt genes was carried out.

\subsection{3'RACE of bkt genes in H. pluvialis}


Three forward primers (Table 1, F1, F2, and F3) were used for 3'RACE. F2 and F3 anneal to the last two exons of $b k t 1$ and $b k t 2$ separated by the last intron of the genes. Coupled with 3'RACE PCR anchor primer, F2 and F3 were used for the first round of 3'RACE. Both bkt1 and bkt2 share the same sequence of primer F1. A nested PCR with F1 and 3'RACE PCR anchor primer generated fragments of about $700 \mathrm{bp}$ in length that may contain the 3'portions of individual bkt genes. The fragments were subcloned into pTZ57R/T vectors. Clones with different inserts were detected by PCR with primers F1 + R1/R2. Of 10 clones analyzed, 5 generated the $500 \mathrm{bp}$ fragment, 4 generated the $348 \mathrm{bp}$ fragment, and 1 generated the $370 \mathrm{bp}$ fragment. The inserts were sequenced, which proved to be the 3 ' termini of bkt1 (bases from 1060 to 1761 in $\underline{\mathbf{X 8 6 7 8 2}}$ ), bkt2 (bases from 1034 to 1662 in D45881), and a similar bkt2 (94\% identity) assigned as bkt3. Figure 3 shows the alignment of the three sequences. Although highly similar, bkt2 and bkt3 represent two distinct genes because in addition to $48 \mathrm{bp}$ differences (out of 635 bp), bkt3 was 22 bp longer than bkt2. The 370 bp band in Fig. 1 (lane 2) is derived from this bkt3.

\subsection{Cloning of the full-length $b k t 3$}

Since $b k t 2$ and $b k t 3$ share highly homologous 3 ' terminal sequence, we predict they also share highly homologous 5 ' terminal sequences. Thus RT-PCR with the reverse primer Rb2 (priming to both $b k t 2$ and bkt3; see Fig. 3) and the forward primer Fb2 (priming to bkt2; see Table 1) may amplify both bkt2 and bkt3 cDNA. RT-PCR with the primers generated $1.7 \mathrm{~kb}$ products. These products were cloned into $\mathrm{pTZ} 57 \mathrm{R} / \mathrm{T}$ vector. Clones containing bkt3 were detected by PCR with primers F1 and R2, which generated 370 bp rather than 348 bp fragments. Positive clones were isolated and sequenced. Sequence data reveals the existence of a full-length $b k t 3$ (GenBank accession no. AY603347). The $b k t 3$ was highly homologous to $b k t 2$ with only 22 
nucleotide differences in their coding region resulting in only two amino acid differences from that of $b k t 2$.

\subsection{Functional analysis of bkt3 in E. coli}

In order to find out whether the bkt3 encodes a functional $\beta$-carotene ketolase, the plasmid pHPb3 (described in Materials and methods) was co-transformed with pACCAR16 $16 \mathrm{crtX}$ or pACCAR25 $\Delta$ crtX respectively into JM109. HPLC analysis of carotenoids from the transformants

is shown in Fig. 4. Compared with pigments extracted from E. coli harboring only the pACCAR16 $\Delta$ crtX which accumulates $\beta$-carotene (Fig. 4B), the E. coli containing the $\mathrm{pHPb} 3$ and pACCAR16 $\Delta$ crtX accumulated canthaxanthin (peak 1) and echinenone (peak 2) in addition to $\beta$ carotene (peak 3). These results indicate that similar to bkt 1 and bkt2, bkt3 encodes an enzyme with activity of converting $\beta$-carotene to echinenone and canthanxanthin (Kajiwara et al., 1995; Lotan and Hirschberg, 1995; Breitenbach et al., 1996). Concordant with the result from Lotan and Hirschberg (1995), no astaxanthin was found in the pigments from E. coli carrying the $\mathrm{pHPb} 3$ and $\mathrm{pACCAR} 25 \Delta \mathrm{crtX}$ (data not shown).

\subsection{Assays of bkt expression by multiple RT-PCR}

The expression of bkt genes under high light intensity $\left(125 \mu \mathrm{mol}\right.$ photons $\left.\mathrm{m}^{-2} \mathrm{~s}^{-1}\right)$, nitrogen limitation, and salt stress was monitored using multiple RT-PCR. The forward primers (F2, F3) used anneal to sequences in the last two exons on both sides of the last intron. Thus potential contaminating genomic DNA did not interrupt the results. RT-PCR generated three expected fragments correspondent to the 3 bkt cDNAs in correct sizes (Fig. 5), which further supports the existence of $3 b k t$ genes. Differential expression of the bkt genes was observed under the stress conditions (Fig. 5). Basal expression of the bkt genes was detected from green flagellate cells 
cultured in the medium without acetate (Fig. 5 lane 1). Low concentration of sodium acetate (14.8 $\mathrm{mM}$ ) enhanced the expression of bkt2 and bkt3 (lane2). Nitrogen deficiency and high light irradiation preferentially induced the expression of bkt1 (lane 3, 4), while high concentration of $\mathrm{NaCl}(100 \mathrm{mM})$ or sodium acetate $(45 \mathrm{mM})$ greatly stimulated the expression of all the three $b k t$ genes (lane 5,6). $\mathrm{FeSO}_{4}$ seemed to have a minor effect on the expression of the bkt genes (lane 7).

\subsection{Assays of bkt expression by real-time RT-PCR}

In order to confirm the results obtained by multiplex RT-PCR, we performed real-time quantitative RT-PCR that allows quantifying the absolute level of the bkt transcripts. Because $b k t 2$ and $b k t 3$ share highly homologous sequence, it is difficult to design absolutely specific primers to each of the genes for real-time quantitative PCR with SYBR ${ }^{\circledR}$ Green detection method. Multiple RT-PCR revealed that the expression patterns of bkt2 and bkt3 are very similar. Therefore the expression of $b k t 2$ and $b k t 3$ was only quantified in combination with one pair of primers annealing to the coding region (Table 1 . Fq2 $+3, \mathrm{Rq} 2+3$ ). PCR of cDNA reversely transcribed from total RNA with the primers (Table 1. Fq1, Rq1; Fq2+3, Rq2+3) gave a single product of the expected size determined by agarose gel electrophoresis and melting curve analysis in each case (data not shown). Serial concentrations of plasmid standards resulted in a reproducible standard curve that was linear from $10^{-7}-10^{0}$ fmol with $\mathrm{R}$ value higher than 0.99 (data not shown). The concentrations of bkt mRNA under different stress conditions are shown in Figure 6. The basal expression levels of bkt2 and bkt3 are higher than that of bkt1. A low concentration of acetate $(14.8 \mathrm{mM})$ could stimulate bkt1-3 expression by about two times. Nitrogen deficiency stimulates bkt1-3 expression by 3 times of that in normal growing cells. High light irradiation $\left(125 \mu \mathrm{mol}\right.$ photons $\left.\mathrm{m}^{-2} \mathrm{~s}^{-1}\right)$ or high sodium acetate concentration $(45 \mathrm{mM})$ 
strongly increased the expression of all the three bkt genes (Fig. 6). Simultaneous application of $\mathrm{Fe}^{2+}$ to high sodium acetate alleviated the increase of the expressions of all the three ketolase genes. No astaxanthin was detectable in the cells treated with $14.8 \mathrm{mM}$ sodium acetate or nitrogen deficiency although the bkt transcripts in these cells increase to 2 to 4 times of that in non-induced cells (Fig. 7). High light irradiation $\left(125 \mu \mathrm{mol} \mathrm{m}^{-2} \mathrm{~s}^{-1}\right)$ increased the expression of bkt genes to 18 times resulting in an accumulation of substantial astaxanthin (about $7 \mathrm{mgg}^{-1}$ dry biomass) in three days (Fig. 7). A higher concentration of sodium acetate (45 mM) greatly increased the bkt transcripts (about 52 fold of basic level) accompanied with an accumulation of about $6 \mathrm{mg}$ astaxanthin per g dry weight (Fig. 7). This result indicated that apart from the $b k t$ transcripts, other factors mediated by high light were important for the yield of astaxanthin.

\section{Discussion}

Green cells of $H$. pluvialis normally generate chloroplast-typical non-keto carotenoids (Boussiba, 2000). Upon stress when green cells are transformed to red cysts, total carotenoids in the cells increased and ketocarotenoids are preferentially formed. This is accompanied by the upregulation of $\beta$-carotene hydroxylase and $\beta$-carotene ketolase genes (Grünewald et al., 2000; Grünewald et al., 2001; Steinbrenner and Linden, 2001). For $\beta$-carotene ketolase, two cDNAs were isolated separately from two different strains of $H$. pluvialis (Kajiwara et al., 1995; Lotan and Hirschberg, 1995). The predicted amino acid sequences of the cDNAs shared about $80 \%$ identity and highly similarity to the bacterial ketolase enzymes (Kajiwara et al., 1995; Lotan and Hirschberg, 1995; Misawa et al., 1995a, b). There is a discrepancy of the existence of bkt genes in H. pluvialis (Sun et al., 1998; Grünewald et al., 2001). In this study, we demonstrated the coexistence of bkt1 and bkt2 in a single strain of $H$. pluvialis (Fig. 1). In addition, a third ketolase 
gene, bkt3, was found in the genome of $H$. pluvialis (Fig. 1, 2, 3, 5). By heterologous expression in E. coli, bkt3 encoded an enzyme with activity similar to bkt1 and bkt2 (Kajiwara et al., 1995; Lotan and Hirschberg, 1995). These results open the question on the functional significance of multiple bkt genes in H. pluvialis.

For the first time, quantitative real-time RT-PCR made it possible to determine the steadystate mRNA levels for the three $\beta$-carotene ketolases in response to stress. By checking the sequences of the primers used in a previous study (Sun et al., 1998), we found that the primers designed according to the sequence of bkt2 (Kajiwara et al., 1995) could amplified the combined transcripts of bkt2 and bkt3 simultaneously. While in another study the primers could only recognize the bkt1 mRNA (Grünewald et al., 2000). Our results revealed basic expression of the three ketolase genes in green un-induced cells (Fig. 5). Sun et al. (1998) also detected the presence of bkt mRNA in green cells in a different strain of $H$. pluvialis. The transcript levels of the $b k t$ genes in normal growing cells and cells cultured with $14.8 \mathrm{mM}$ sodium acetate or induced by nitrogen deficiency were below a threshold value necessary for astaxanthin biosynthesis. As a result, no astaxanthin was detected in the cells under the cultured conditions. When the transcript levels of the bkt genes reached $1.6 \times 10^{-6} \mathrm{fmol} / \mathrm{ng}$ total RNA, substantial amounts of astaxanthin were produced as in the case of the alga cells treated with high light irradiation or high salt stress (Fig. 7).

Astaxanthin is located in cytoplasmic lipid globules (Johnson and An, 1991) while its direct precursor $\beta$-carotene is synthesized in chloroplast (Grünewald et al., 2000). There is no clear indication of a transit sequence in bkt1, bkt2 and bkt3. The ketolase was detected in both cytoplasm and chloroplast (Grünewald et al., 2000). It is not possible to gain information about 
the spatial distribution of the three ketolase proteins at present. The major difference of the individual bkt genes was revealed in their response to stress. The expression of all the bkt genes were up-regulated under stress but to different levels. The expression level of bkt1 was even higher than that of the combined amount of bkt2 and bkt3 (Fig. 6) when the alga cells were treated with high light irradiation or sodium acetate stress. $\mathrm{Fe}^{2+}(450 \mu \mathrm{M})$ alleviated the expression of the bkt genes and the yield of astaxanthin induced by sodium acetate $(45 \mathrm{mM})$. This negative effect was confirmed at the messenger level of the $\beta$-carotene hydroxylase and the formation of astaxanthin in the same alga (Steinbrenner and Linden, 2001). Sodium chloride had a similar effect as sodium acetate in inducing the expression of the three genes (Fig. 5), supporting the interpretation that sodium acetate initiates a salt stress as sodium chloride (Boussiba, 2000; Steinbrenner and Linden, 2001).

In conclusion, $H$. pluvialis contains three different $b k t$ genes which are differential regulated by a variety of environmental factors. The existence of multiple bkt genes in $H$. pluvialis and their high expression upon stress conditions make it easy to reach a threshold of total bkt mRNA that is essential for the biosynthesis of large amounts of astaxanthin.

\section{Acknowledgements}

We are grateful to Drs Anderson Wong, Wendy Ko and Keith Fan for technical assistance. This work was supported partially by a grant from the Research Grants Council of Hong Kong, and the Outstanding Young Researcher Award of the University of Hong Kong. 


\section{References}

Boussiba, S., 2000. Carotenogenesis in the green alga Haematococcus pluvialis: cellular physiology and stress response. Phys. Plant. 108, 111-117.

Breitenbach, J., Misawa, N., Kajiwara, S., Sandmann, G., 1996. Expression in Escherichia coli and properties of the carotene ketolase from Haematococcus pluvialis. FEMS Microbiol. Lett. $140,241-246$.

Cunningham, F.X., Gantt, E., 1998. Genes and enzymes of carotenoid biosynthesis in plants. Ann. Rev. Plant Phys. Plant Mol. Biol. 49, 557-583.

Gorman, D.S., Levine, R.P., 1966. Cytochrome $f$ and plastocyanin: their sequence in the photoelectric transport chain. Proc. Natl. Acad. Sci. U.S.A. 54, 1665-1669.

Grünewald, K., Hirschberg, J., Hagen, C., 2001. Ketocarotenoid biosynthesis outside of plastids in the unicellular green alga Haematococcus pluvialis. J. Biol. Chem. 276, 6023-6029.

Grünewald, K., Eckert, M., Hirschberg, J., Hagen, C., 2000. Phytoene desaturase is localized exclusively in the chloroplast and up-regulated at the mRNA level during accumulation of secondary carotenoids in Haematococcus pluvialis (Volvocales, Chlorophyceae). Plant Physiol. 122, 1261-1268.

Hagen, C., Braune, W., Greulich, F., 1993. Functional aspects of secondary carotenoids in Haematococcus lacustris (Girod) Rostafinski (Volvocales): IV. Protection from photodynamic damage. J. Photochem.Photobiol. 20, 153-160.

Johnson, E.A., An, G.H., 1991. Astaxanthin from microbial sources. Crit. Rev. Biotechnol. 11, 297-326.

Johnson, E.A.,Schroeder, W.A., 1996. Biotechnology of astaxanthin production in Phaffia rhodozyma. In Biotechnology for improved foods and Flavors (eds Takeda G.R, Teranishi R. and Williams P.J.), pp 39-50. American Chemical Society. 
Kajiwara, S., Kakizono, T., Saito, T., Kondo, K., Ohtani, T., Nishio, N., Nagi, S., Misawa, N., 1995. Isolation and functional identification of a novel cDNA for astaxanthin biosynthesis from Haematococcus pluvialis, and astaxanthin synthesis in Escherichia coli. Plant Mol. Biol. 29, 343-352.

Kobayashi, M., Kakizono, T., Yamaguchi, K., Nishio, N., Nagai, S., 1992. Growth and astaxanthin formation of Haematococcus pluvialis in heterotrophic and mixotrophic condition. J. Ferment. Bioengi. 74, 17-20.

Kobayashi, M., Kakizono, T., Nagai, S., 1993. Enhanced carotenoid biosynthesis by oxidative stress in acetate-induced cyst cells of a green unicellular alga, Haematococcus pluvialis. Appl. Env. Microbiol. 59, 867-873.

Kobayashi, M., Kakizono, T., Nishio, N., Nagai, S., Kurimura, Y., Tsuji, Y., 1997. Antioxidant role of astaxanthin in the green alga Haematococcus pluvialis. Appl. Microbiol. Biotechnol.. 48, 351-356.

Linden, H., 1999. Carotenoid hydroxylase from Haematococcus pluvialis: cDNA sequence, regulation and functional complementation. Biochem. Biophys. Acta 1446, 203-212.

Lotan, T., Hirschberg, J., 1995. Cloning and expression in Escherichia coli of the gene encoding ß-C-4-oxygnease that converts $\beta$-carotene to the ketocarotenoid canthaxanthin in Haematococcus pluvialis. FEBS Lett. 364, 125-128.

Meyers, S.P., 1994. Developments in world aquaculture, feed formulations, and role of carotenoids. Pure Appl. Chem. 66, 1069-1076.

Misawa, N, Nakagawa, M., Kobayashi, K., Yamano, S., Izawa, Y., Nakamura, K., Harashima, K., 1990. Elucidation of the Erwinia uredovora carotenoid biosynthetic-pathway by functional analysis of gene products expressed in Escherichia coli. J.Bacteriol. 172, 67046712. 
Misawa, N., Kajiwara, S., Kondo, K, Yokoyama, A., Satomi, Y., Saito, T., Miki, W., Ohtani, T., 1995a. Canthaxanthin biosynthesis by the conversion of methylene to keto groups in a hydrocarbon ß-carotene by a single gene. Biochem. Biophys. Res. Com. 209, 867-876.

Misawa, N., Satomi, Y., Kondo, K., Yokoyama, Y., Kajiwara, S., 1995b. Structure and functional analysis of a marine bacterial carotenoid biosynthesis gene cluster and astaxanthin biosynthetic pathway proposed at the gene level. J. Bacteriol. 177, 6575-6584.

Sambrook, J., Fritsch, E.F., Maniatis, T., 1989. Molecular Cloning: A Laboratory Manual. Cold Spring Harbor Laboratory Press, Cold Spring Harbor, NY.

Steinbrenner, J., Linden, H., 2001. Regulation of two carotenoid biosynthesis genes coding for phytoene synthase and carotenoid hydroxylase during stress-induced astaxanthin formation in the green alga Haematococcus pluvialis. Plant Physiol. 125, 810-817.

Steinbrenner, J., Linden, H., 2003. Light induction of carotenoid biosynthesis genes in the green alga Haematococcus pluvialis: regulation by photosynthetic redox control. Plant Mol. Biol. $52,343-356$.

Stewart. C.N.. Via, L.E., 1993. A rapid CTAB DNA isolation technique useful for rapid fingerprinting and other PCR amplifications. BioTechn. 14, 748-751.

Sun, Z, Cunningham, F.X., Gantt, E., 1998. Differential expression of two isopentenyl pyrophosphate isomerases and enhanced carotenoid accumulation in a unicellular chlorophyte. Proc. Natl. Acad. Sci. U.S.A. 95, 11482-11488.

Yong ,Y.Y.R., Lee, Y.K., 1991. Do carotenoids play a photoprotective role in the cytoplasm of Haematococcus lacustris (Chlorophyta)? J. Phycology 30, 257-261.

Yuan, J.P., Chen, F., 1998. Chromatographic separation and purification of trans-astaxanthin from the extracts of Haematococcus pluvialis. J. Agr. Food Chem. 46, 3371-3375. 
TABLE 1.Primer sets and PCR product characteristics

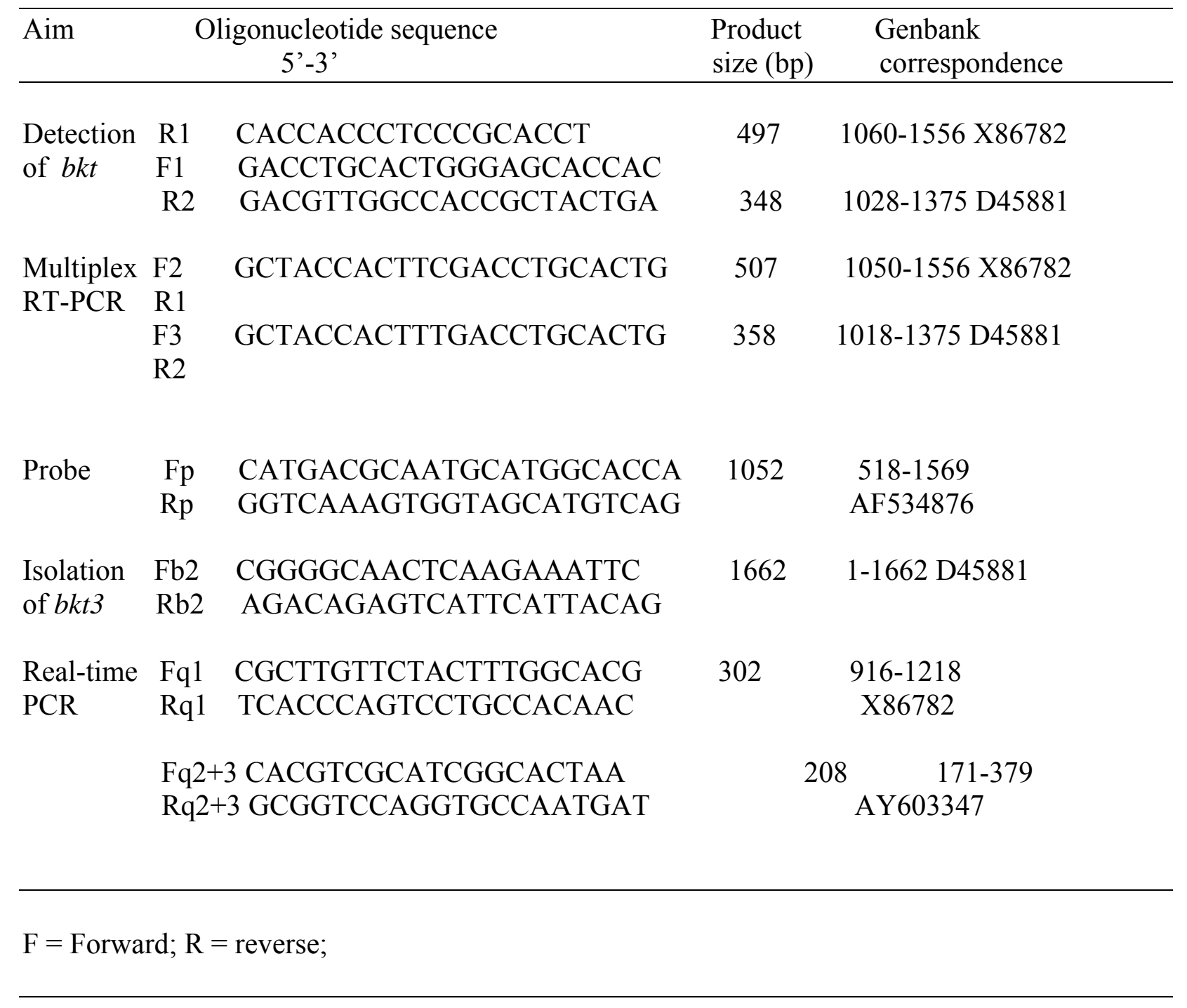




\section{Figure legends}

Fig. 1. PCR detection of bkt genes in H. pluvialis NISE 144. The bkt specific PCR product was separated on a $1.5 \%$ agarose gel. PCR was performed using primers specific to bkt2 $(1,2)$ or bkt1 $(3,4)$ and DNA templates of plasmid containing the bkt2 (1), bkt1 (3), or H. pluvialis genomic DNA $(2,4)$. M, 100 bp DNA ladder plus (Fermentas).

Fig. 2. Southern analysis of genomic DNA from $H$. pluvialis NISE 144 . Genomic DNA ( $8 \mu \mathrm{g})$ was digested with SacI (lane 2), or KpnI + SalI (lane 3). Plasmids (400 pg) containing the bkt2 gene cut by SacI (lane 1) or EcoRI (releasing the bkt2, lane 4) were used as positive controls.

Fig. 3. Nucleotide sequence alignment of 3' termini of bkt genes from H. pluvialis NISE 144. The sequences are aligned by using the program CLUSTAL X. Bold nt sequences correspond to forward primers, bold and italic nt sequences correspond to reverse primers used in the detection of bkt genes.

Fig. 4. HPLC analysis of carotenoid pigments extracted from E. coli cells carrying plasmid pACCAR16 $\Delta$ crtX and pHPb3 (A) or pACCAR16 $\Delta \mathrm{crtX}$ (B). 1-canthaxanthin; 2-echinenone; 3-ßcarotene.

Fig. 5. Analysis of the differential expression of $b k t$ gene in H. pluvialis cells using RT-PCR. RTPCR was performed using RNAs from cells grown in a medium without sodium acetate (1), with 
$14.8 \mathrm{mM}$ sodium acetate (2), nitrogen deficiency (3), under high light irradiation (4), with 100 $\mathrm{mM} \mathrm{NaCl}$ (5), with $45 \mathrm{mM}$ sodium acetate (6), or with $450 \mu \mathrm{M} \mathrm{FeSO}_{4}$ (7) for $16 \mathrm{~h}$. The bkt specific PCR product was separated on a $2.5 \%$ agarose gel (A), along with internal controls (actin) amplification (B). M, 100 bp DNA ladder plus (Fermentas).

Fig. 6. Expression of $b k t 1$ ( $\square$ ) and $b k t 2+3$ ( $\mathbf{a})$ detected by quantitative real-time PCR. Real-time PCR was carried out with cDNA from total RNA isolated from H. pluvialis cells induced by 14.8 $\mathrm{mM}$ sodium acetate (Lac), nitrogen deficiency (ND), high light irradiation (HL), $45 \mathrm{mM}$ sodium acetate (Hac), and $45 \mathrm{mM}$ sodium acetate with $450 \mu \mathrm{M} \mathrm{Fe} \mathrm{e}^{2+}$ for $16 \mathrm{~h}$. AcDe represents samples from normal growing cells cultured with no sodium acetate. Each value is the mean $\pm \mathrm{SE}(\mathrm{n}=2)$.

Fig. 7. Expression of bkt1-3 (A) and astaxanthin accumulation (B) after growth under various stress condition. Gene expression was quantified by real-time PCR after $16 \mathrm{~h}$ of induction. Astaxanthin was quantified after $72 \mathrm{~h}$ of induction. 
Fig. 1.

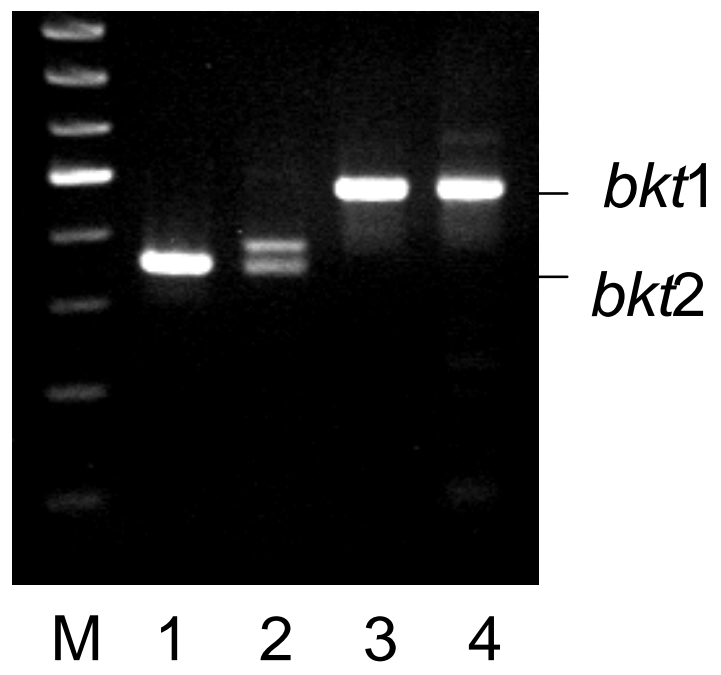


Fig. 2.

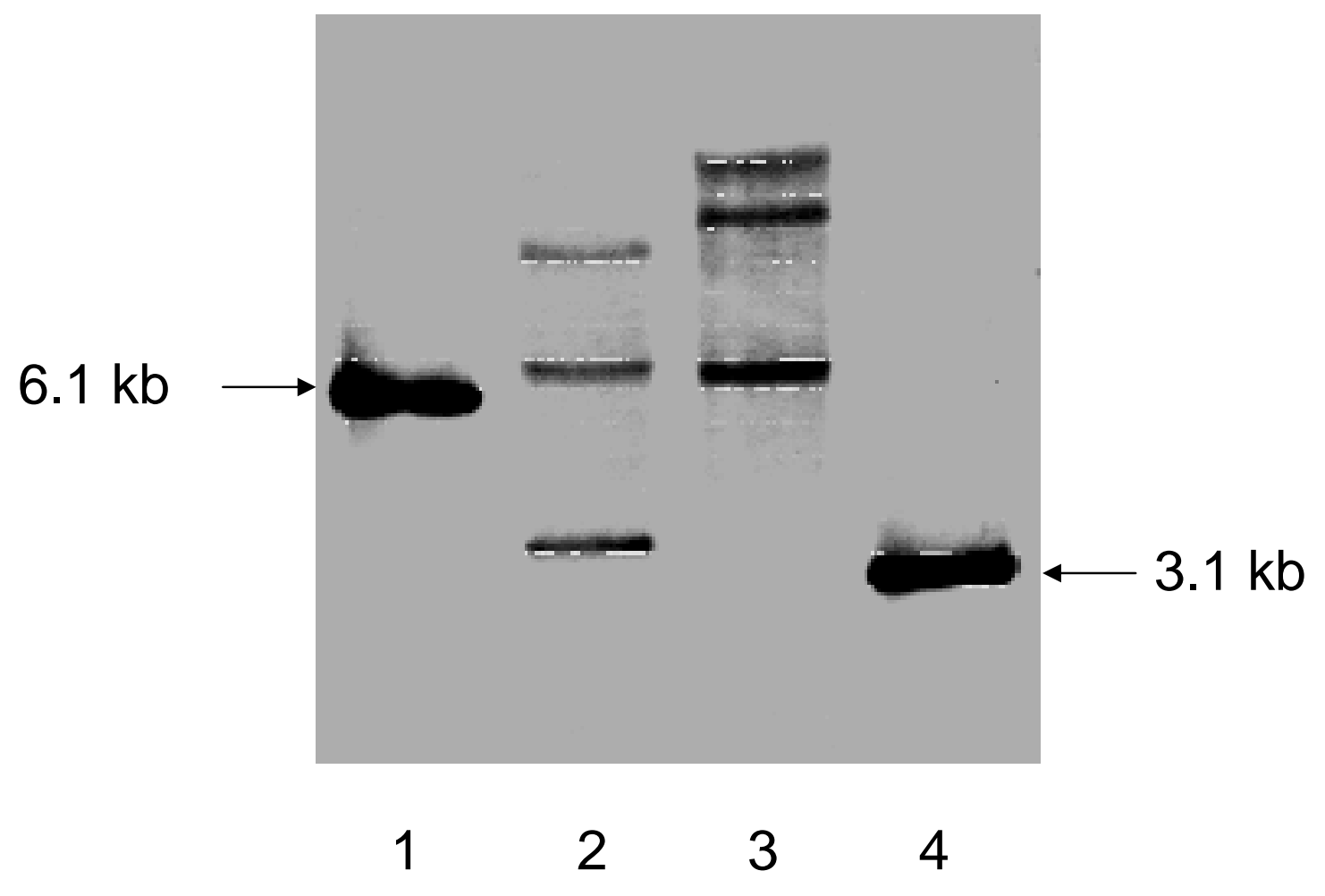


Fig. 3.

bkt 3

bkt2

bkt 1

bkt3

bkt2

bkt 1

bkt 3

bkt2

bkt 1

bkt3

bkt2

bkt 1

bkt 3

bkt2

bkt 1

bkt3

bkt2

bkt 1

bkt 3

bkt2

bkt 1

GACCTGCACTGGGAGCACCACAGGTGGCCCTTTGCTCCCTGGTGGCAGCTGCCCCACTGC GACCTGCACTGGGAGCACCACAGGTGGCCCTTTGCCCCCTGGTGGCAGCTGCCCCACTGC GACCTGCACTGGGAGCACCACCGCTGGCCCTTCGCCCCCTGGTGGGAGCTGCCCAACTGC ************************ * ********** ** *********** $* * * * * * * * * * * * * * *$

CGCCGCCTGTCGGGGCGTGGCCTGGTGCCTGCCTTGGCATGACCTGGTCCCTCCGCCAGT CGCCGCCTGTCCGGGCGTGGCCAGGTGCCTGCCTTGGCATGACCTGGTCCCTCCGCTGGT CGCCGCCTGTCTGGCCGAGGTCTGGTTCCTGCCTAGCTGGACACTGCAGTGGGCCCTGCT $* * * * * * * * * * * * * * * * * * * * * * * * * * * * * *$ $* * *$ $* * *$

GTGACCCGGCGTCTGCATAAGAGTGTCATGCTACAGGGTGATGCGGCCAGTGGCGGCGCA G--ACCCAGCGTCTGCACAAGAGTGTCATGCTACAGGGTGCTGCGGCCAGTGGCAGCGCA GCCAGCTGG-GCATGCA---GGTTGTGGCAGGACTGGGTGAGGTGAAGAGCTGCAG-GCG $* * * * * * * * * * \quad * \quad * * * \quad * * * * * * * \quad * * \quad * * \quad * * * * *$

GTGCACTCTTTGAGCCTGTATGGGGCTACTGCTGTGCCACTGAGCACT-GGGTTTGCCAC GTGCACTCTC--AGCCTGTATGGGGCTACCGCTGTGCCACTGAGCACT-GGGCATGCCAC CTGCTGCCGGACACACTGCATGGGCTGCCCTGTGTACCTGTCGCCACTAGGGGAGGGGGC $* * * * * * * * * * * * * \quad * \quad * * * * * * * \quad * * * * * * * \quad * \quad *$

TG-AGC-ACTGGGTTTGCCACTGAGCACTGGGCGTGCTACTGATGTCTGGAAGTGTAGGC TG-AGC-ACTGGGCGTGCTACTGAGCAATGGGCGTGCTACTGAGCAATGGGCGTGC---T TGTAGCTGCCAAGCATGCCCCACGGATGAAGCTGTG-TAGTGGTGAA-GGGAGTACACCC

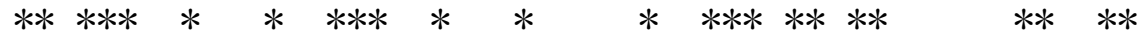

AGTGGCAGACTGCAGTGTAGGCAGTGGCTGAGATGGAGTTCTGATGCAGTCAGTGATGGA ACTGACA-A--------TGGGCG-----TGCTACTGGGGTCTGGCAGTGGCTAGGATGGA ACAGGCTAACACCCTTGCAGGAGTTGTCTTGCATGGGGAGAGGTGGTGGGCGGTGTAGAT $* * * * * \quad * * \quad * \quad * \quad * * * \quad * \quad * \quad * * \quad * \quad *$

GTTCTGA-TGCAG-TCAGTA-----------GCGGTGGCCAACGTCATGTGGATGGTG-GTT-TGA-TGCAT- TCAGTA-----------GCGGTGGCCAACGTCATGTGGATGGTG-GCTACGACTGTATCTTAGTATCTTAGGGGGAGCGACACTTAGTGCTGGGCAGGCAACGCC $* * * * * * * * * * * *$ $* * *$ * $* * * * *$ 


\begin{tabular}{|c|c|}
\hline bkt3 & ----GGAGTGCTGAGGGGGGTTAGGCAGCCGGCATTTGAGAGGGCTAAGTTATAAATCGC \\
\hline bkt2 & ----GAAGTGCTGAGGGGT-TTAGGCAGCCGGCATTTGAGAGGGCTAAGTTATAAATCGC \\
\hline \multirow[t]{2}{*}{ bkt1 } & CTGCAAGGTGCCGACACAAGCTAGG-AGCTGACGTGGACGAGGAGTCAGTGGCAGGCAGA \\
\hline & $* * * * * * * * * * *$ \\
\hline bkt3 & --ATGCTGCTCATGCGCACATATCTGCACACAGCCAGGGGAATCC--CTTCGA-GAGTGA \\
\hline bkt2 & --ATGCTGCCCATGCGCACATATCTGCACACAGCCAGGGAAATCCT-CTTCGA-GAGTGA \\
\hline \multirow[t]{2}{*}{ bkt1 } & TGAAGAGGTGCGGGAGGGTGGTGCCACACCCACTGGGCAAGACCATGCTGCAATGCCTGG \\
\hline & 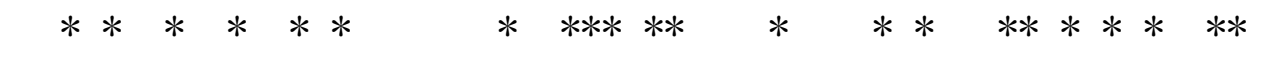 \\
\hline bkt3 & TTATGGGACACTTGTAT-----TGGTT---TCGTGCTATTGTTTTATTCAGCACCAGCACT \\
\hline$b k+2$ & TTATGGGACACTTGTAT-----TGGTT---TCGTGCTATTGTTTTATTCAGCACCAGCACT \\
\hline \multirow[t]{2}{*}{ bkt1 } & CGGTGTGGCAGTGAGAGCTGCGTGATTAACTGGGCTATGGATTGTTTGAGCAGTCTCACT \\
\hline & 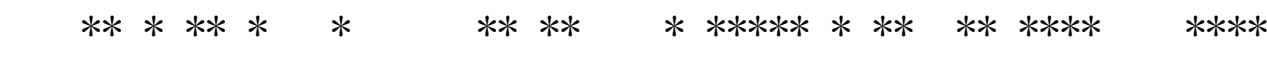 \\
\hline$b k+3$ & TAGT----GAGGGTGAGAGCAGGGTGGTGAGAGTGGAGTGAGTGAGCATGAACCTGGTCA \\
\hline$b k t 2$ & TAGT----GAGGGTGAGAGCAGGGTGGTGAGAGTGGAGTGAGTGAGCATGAACCTGGTCA \\
\hline \multirow[t]{2}{*}{ bkt1 } & TATTCTTTGATATAGACACTGGTCAGGCAGGTCAGGAGAGAGTGAGTATGAACAAGTTGA \\
\hline & 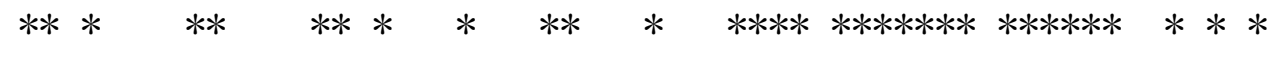 \\
\hline bkt3 & GCAA-GGTGAAAAGTCTGTA-ATGAATGACTCTGTCT--------------- \\
\hline bkt2 & GCGA-GGT-AACAGCCTGTA-ATGAATGACTCTGTCT--------------- \\
\hline \multirow[t]{2}{*}{ bkt1 } & GAGGTGGTGCGCTGCCCCTGCGCTTATGAAGCTGTAACAATAAAGTGGTTC \\
\hline & $* \quad * * * \quad * * * \quad * \quad \quad * * * * \quad * * * *$ \\
\hline
\end{tabular}


Fig. 4
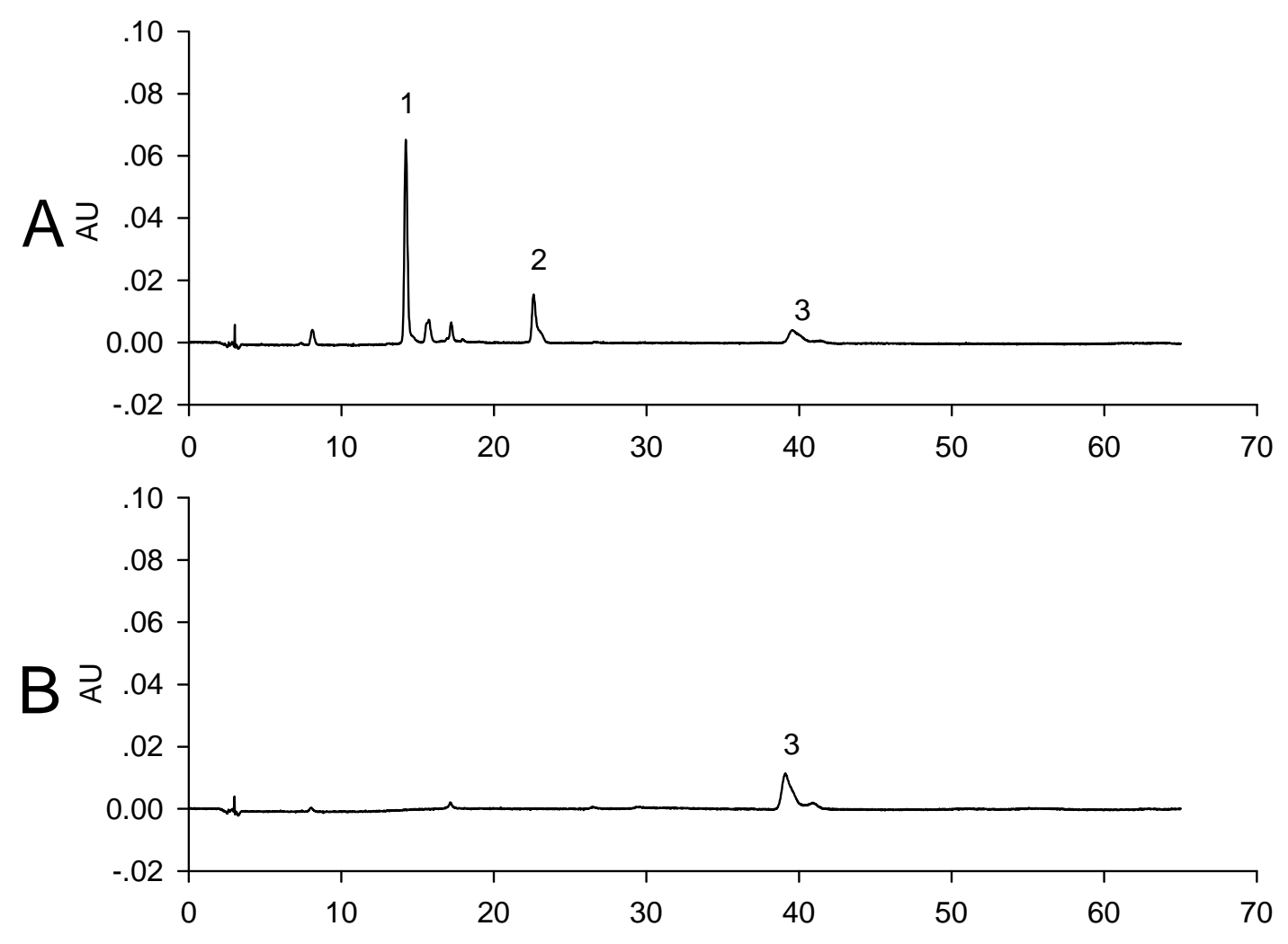

Time (min) 
Fig. 5.

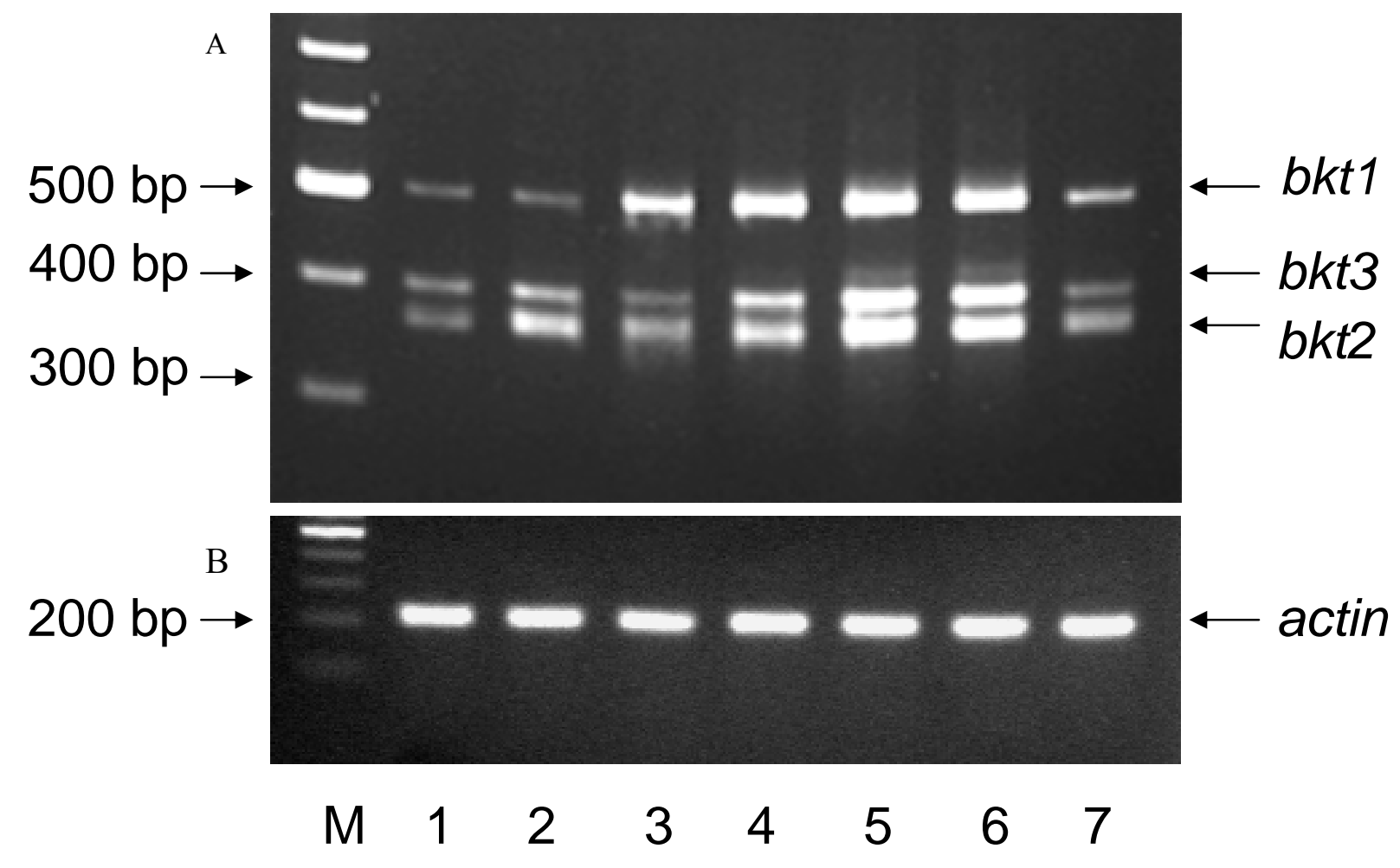


Fig. 6.

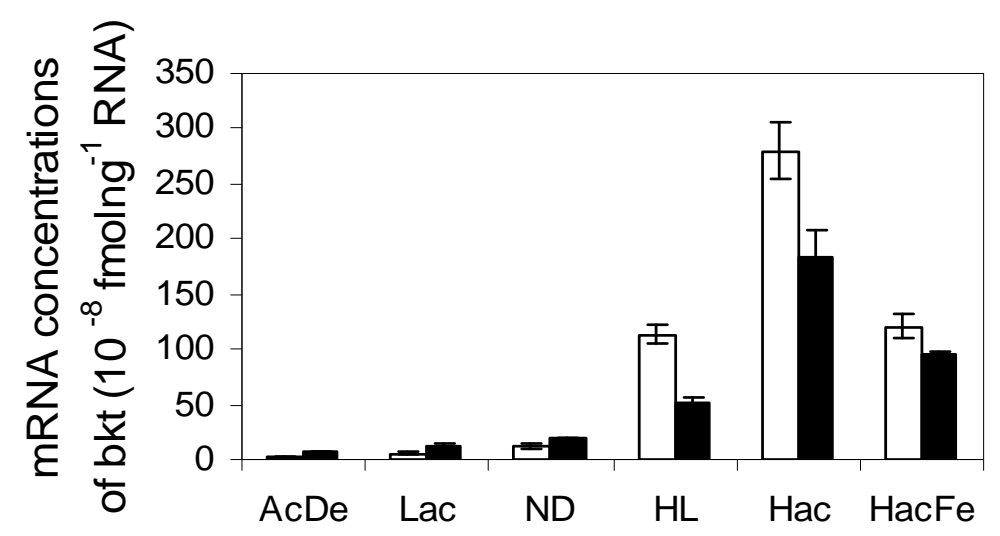


Fig. 7.
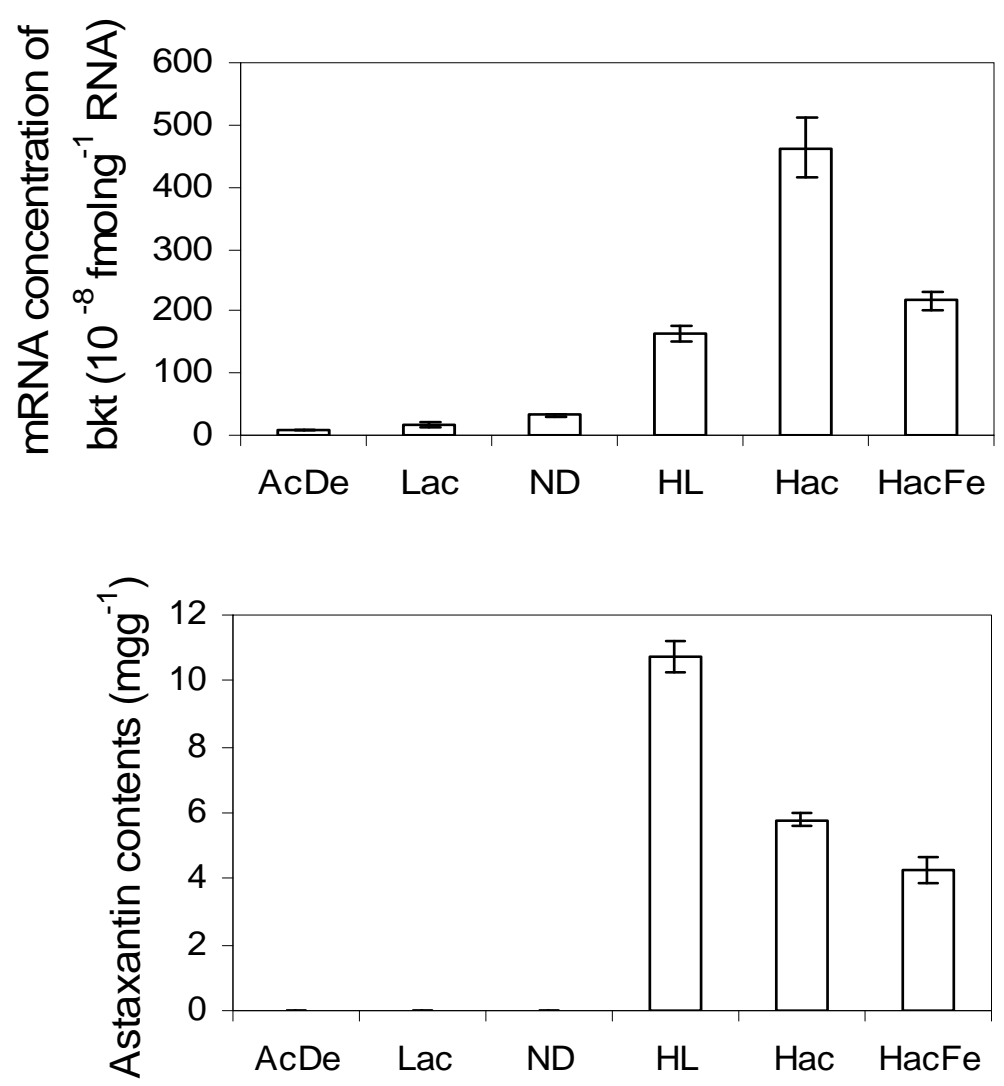